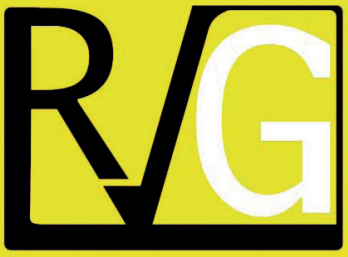

Julio - Septiembre, 2021

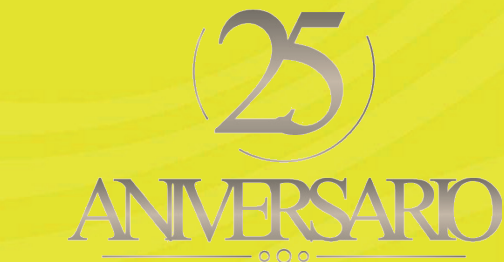

ANMERSARO
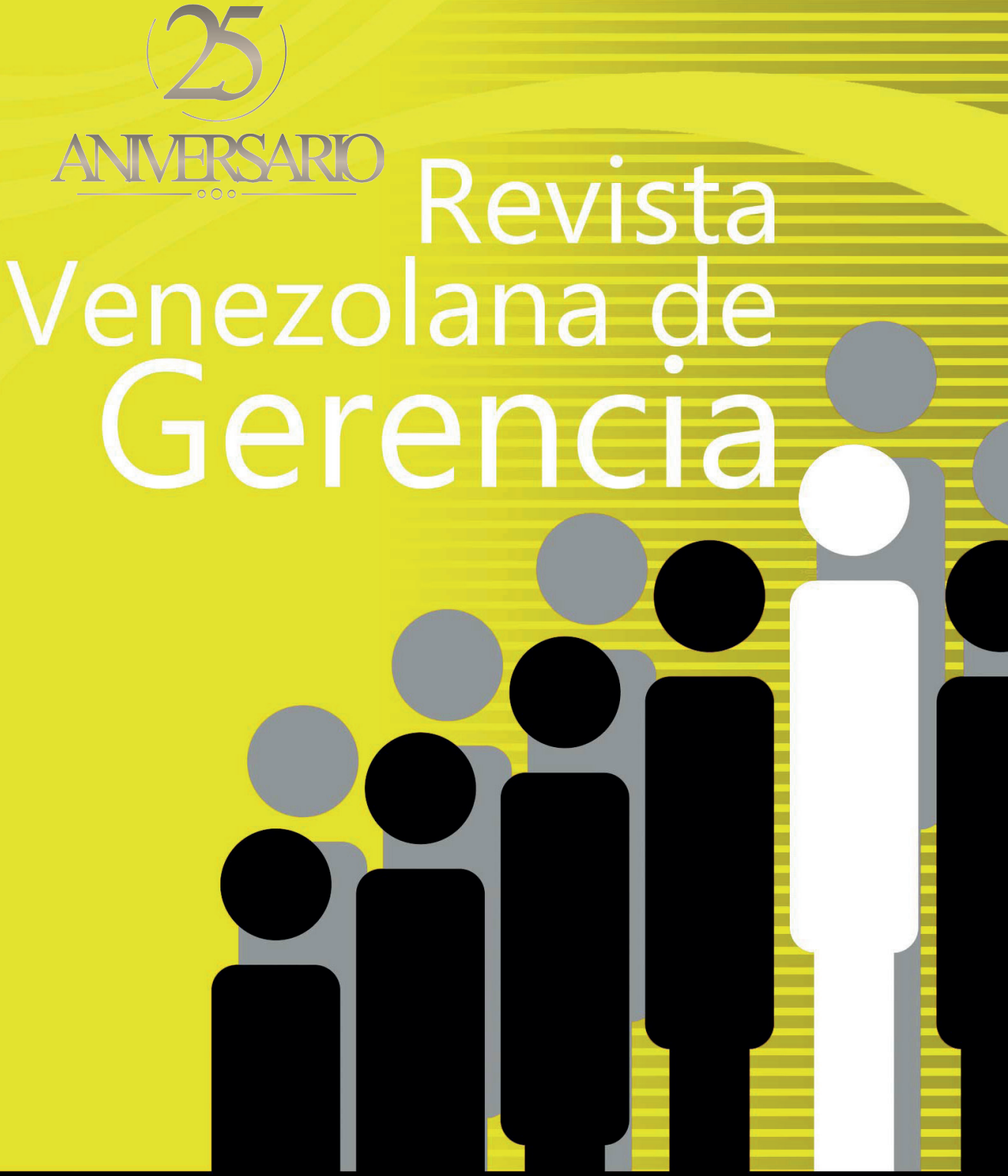

UNIVERSIDAD DEL ZULIA (LUZ)

Facultad de Ciencias Económicas y Sociales Centro de Estudios de la Empresa

ISSN 1315-99

Esta obra está bajo una licencia de Creative Comm Reconocimiento-NoComercial-Compartirlgual 3.0 Unpo http://creativecommons.org/licenses/by-nc-sa/3.0/deed.es 
COMO CITAR: Villalobos Rodríguez, G., Moraga López, G., Guevara Portuguez, Ma. J., y Araya-Castillo, L. (2021). Desempleo juvenil: contribuciones para su disminución desde el emprendimiento. Revista Venezolana de Gerencia (RVG), 26(95), 758-775. https://doi.org/10.52080/rvgluz.27.95.20
Universidad del Zulia (LUZ)

Revista Venezolana de Gerencia (RVG)

Año 26 No. 95 Julio-Septiembre 2021, 758-775

ISSN 1315-9984 / e-ISSN 2477-9423

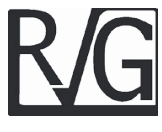

\title{
Desempleo juvenil: contribuciones para su disminución desde el emprendimiento
}

\author{
Villalobos Rodríguez, Gerardo* \\ Moraga López, Geannina** \\ Guevara Portuguez, María José*** \\ Araya-Castillo, Luis****
}

\section{Resumen}

La promoción de nuevos emprendimientos es uno de los pilares básicos y eje estratégico para generar inclusión social, oportunidades laborales, innovación, desarrollo económico y social en las economías latinoamericanas; siendo para muchos jóvenes latinoamericanos la única opción de obtener un ingreso decente. Los niveles de desempleo juvenil en el año 2020, en gran parte de las economías de la región, superan el $20 \%$, por lo que resulta urgente identificar alternativas de generación de empleo, que permitan mejorar la calidad de vida de la población en general y de los jóvenes en particular. El análisis se basa en la revisión documental de las fuentes técnico científico relacionadas con desempleo juvenil y el emprendimiento como alternativa al fenómeno; especialmente con datos de los reportes recientes (entre los años 2018 al 2021). El desempleo en la población joven es un problema generalizado que afecta a los países de la región latinoamericana, lo cual se asentó con la pandemia del COVID-19. EI emprendimiento es una herramienta clave para atender directamente la problemática del desempleo y los creados por personas jóvenes están generando fuentes de empleo en los países latinoamericanos

Palabras clave: Desempleo; desempleo juvenil; trabajo; emprendimiento; educación.

Recibido: 01.02.21

Aceptado: 23.06 .21

* Doctor en Ciencias Empresariales de la Universidad Fidélitas de Costa Rica. Maestría en Administración de Negocios, Mercadeo y Finanzas, Universidad de Costa Rica (UCR). Especialidad en Responsabilidad Social Corporativa, Universidad de Buenos Aires, Argentina. Académico de la Vicerrectoría de Investigación de la Universidad Nacional de Costa Rica. Correo electrónico: gerardo.villalobos.rodriguez@una.cr https://orcid. org/0000-0002-3104-7472

** Máster en Gerencia de Proyectos. Asesora de proyectos de investigación e innovación, Vicerrectoría de Investigación, Universidad Nacional, Costa Rica. Correo electrónico: gmoraga@una.ac.cr https://orcid. org/0000-0002-8221-8489

*** Bachiller en Sociología y estudiante de Licenciatura en Sociología, Universidad Nacional, Costa Rica, maria. guevara.portuguez@est.una.ac.cr ; https://orcid.org/0000-0002-9392-8195

*** PhD in Management Sciences, ESADE Business School; Doctor en Ciencias de la Gestión, Universidad Ramon Llull; Doctor en Empresa, Universidad de Barcelona. Director de Postgrado y Educación Continua, Profesor Asociado, Facultad de Economía y Negocios, Universidad Andrés Bello, Chile. Correo electrónico: luis.araya@unab.cl https://orcid.org/0000-0001-7574-3907 


\title{
Evidence of the contribution of entrepreneurship to the reduction of youth unemployment
}

\begin{abstract}
The promotion of new ventures is one of the basic pillars and strategic axes to generate social inclusion, job opportunities, innovation, economic and social development in Latin American economies; being for many young Latin Americans the only option to obtain a decent income. The levels of youth unemployment in 2020 , in a large part of the economies of the region, exceed $20 \%$, so it is urgent to identify alternatives for generating employment, which allow improving the quality of life of the population in general and of young people. The analysis is based on the documentary review of technical scientific sources related to youth unemployment and entrepreneurship as an alternative to the phenomenon; especially with data from recent reports (between the years 2018 to 2021). Unemployment in the young population is a generalized problem that affects the countries of the Latin American region, which settled with the COVID-19 pandemic. Entrepreneurship is a key tool to directly address the problem of unemployment and those created by young people are generating sources of employment in Latin American countries.
\end{abstract}

Keywords: Unemployment, youth unemployment, work, entrepreneurship, education.

\section{Introducción}

De acuerdo con la Organización Internacional del Trabajo (OIT), la crisis derivada por la propagación del virus SARS-CoV-2 ha tenido un impacto negativo sobre los mercados laborales de América Latina y el Caribe, donde millones de personas han perdido sus empleos y sus ingresos debido a la caída de la actividad económica (OIT, 2020b).

La pandemia evidencia con mayor claridad las desigualdades existentes en los países latinoamericanos, lo que se ve reflejado en el aumento de la pobreza y otras desigualdades sociales. Además, muestra los grandes desafíos del trabajo en condiciones de informalidad, con bajos ingresos, sin protección social y sin acceso a la salud.

La población joven está entre los que más padecen las consecuencias sociales y económicas de la pandemia en la región, y harán frente a los efectos de esta en los próximos años de su vida laboral, corriendo el riesgo de pasar a constituir lo que la OIT (2020a) denomina una "generación de confinamiento".

La misma entidad establece que la persistencia de la COVID-19 está retrasando la recuperación económica en prácticamente todos los países de la región. Los efectos de las medidas para enfrentar la pandemia, como el confinamiento, ha provocado la quiebra $y$ el cierre de numerosas empresas locales. Esto se ve reflejado en la pérdida de ingresos entre los trabajadores de 
Villalobos Rodríguez, Gerardo; Moraga López, Geannina;

Guevara Portuguez, María José y Araya-Castillo, Luis

Desempleo juvenil: contribuciones para su disminución desde el emprendimiento

todas las edades y la pérdida de trabajos.

La OIT (2020a) ha estimado que, a nivel mundial, 4 de cada 10 personas jóvenes se desempeñan en los sectores de servicios y comercio, los cuales resultaron gravemente afectados por la COVID-19. Relacionado con el planteamiento anterior, la Comisión Económica para América Latina y el Caribe [CEPAL] (2020) señala que la crisis económica y social derivada del COVID-19 es la más fuerte que ha experimentado la región en varias décadas, y ha puesto de manifiesto las debilidades estructurales de las economías. Se resalta que esta pandemia por el SARS-CoV-2 ha llevado a la economía mundial a la peor recesión desde la Segunda Guerra Mundial, y se registra el mayor porcentaje de países $(90 \%)$ que experimentan una recesión de manera simultánea, desde que se tienen estimaciones.

Ante esta situación, la OIT (2020b) establece un marco general de políticas basada en cuatro pilares, el estímulo de la economía y el empleo a través de incentivos fiscales; la creación de nuevos emprendimientos; el apoyo a las empresas establecidas, los empleos y los ingresos; y la protección a los trabajadores en el lugar de trabajo y la búsqueda de soluciones mediante el diálogo social.

Dos años atrás, la misma OIT (2019) planteaba que el estímulo al emprendimiento y a la creación de nuevas empresas, ha sido históricamente el principal contribuyente de nuevos empleos en la región latinoamericana y en el mundo. Bajo esta perspectiva, en el contexto actual se requiere de nuevas condiciones para el fomento y creación de emprendimientos que a su vez sean fuentes potentes y dinamizadores de empleos.
En este artículo se reúnen e interpretan evidencias de cómo el emprendimiento contribuye con la disminución del desempleo y con énfasis en la población joven, en la región latinoamericana.

Desde el plano metodológico, se aborda como una investigación descriptiva sobre el emprendimiento como alternativa para el desempleo juvenil, basado en fuentes secundarias con datos e información de organizaciones de Derecho Internacional Público, como por ejemplo la Organización para la Cooperación y el Desarrollo Económico [OCDE], la Comisión Económica para América Latina y el Caribe [CEPAL], el Global Entrepreneurship Monitor [GEM] (2018, 2019, 2020 y 2021) y la Organización Internacional del Trabajo [OIT].

Además, se realizó una búsqueda de la literatura en bases de datos indexadas como Scopus, Business Source Ultimate y Sciencedirect, mediante palabras clave como "desempleo juvenil" y "emprendimiento". Dentro de las fuentes seleccionadas y analizadas, se encuentran reportes recientes, entre los años 2018-2021, las cuales buscan dar respuesta a la siguiente pregunta de investigación ¿contribuye el emprendimiento a la disminución del desempleo juvenil?

\section{Desempleo juvenil: repaso teórico}

El aumento del desempleo es un fenómeno estructural derivado del deterioro de las actividades económicas de un país, como dimensión de este, el desempleo juvenil se visualiza como uno de los grandes retos y desafíos a atender en la región latinoamericana. Tal y como se evidencia en este artículo, 
en los últimos años, el desempleo juvenil se aceleró en forma significativa en prácticamente todos los países de la región latinoamericana, generando frustración y desmotivación, pero principalmente convirtiéndose en caldo de cultivo para el aumento de la pobreza y otras problemáticas sociales.

La pandemia de la COVID-19 contribuyó a elevar las cifras de desempleo juvenil en prácticamente todos los países de la región. Klisberg (2007) plantea que las recesiones y crisis internacionales de los últimos años, el aumento de la competitividad de las empresas, los permanentes cambios tecnológicos y las incertidumbres generadas por cambios legislativos, son sólo algunas de las explicaciones para que los niveles de desempleo sean mayores año con año.

Las personas jóvenes representan a un grupo de la población fuertemente afectada por el desempleo, lo que intensifica su vulnerabilidad económica (Brunner et al., 2020). De modo que, el empleo para las personas jóvenes se muestra como un desafío político, porque las expectativas traducidas en desaliento y frustración debilitan la estabilidad de las sociedades (Klisberg, 2007).

Klisberg (2007) analiza los indicadores de escolaridad de la mayoría de los países de Latinoamérica, evidenciando una paradoja: la población joven forma parte de la generación más educada de los últimos tiempos. Un buen porcentaje ha ido a la universidad y tiene expectativas sobre su propio futuro en el mundo del trabajo, sin embargo, muchos se encuentran desempleados. Se evidencia una desaceleración en el crecimiento del empleo mundial, el crecimiento del desempleo y del subempleo, y, por consiguiente, de la desilusión, como los principales factores que afectan a los jóvenes en la última década. El resultado es que la juventud se enfrenta a un progresivo déficit de oportunidades de trabajo y altos niveles de incertidumbre económica y social.

Se han tipificado muchas causas en los ámbitos académicos y científicos, como detonadoras o que contribuyen al aumento del desempleo juvenil. Por ejemplo, para Smyth et al. (2003) el contexto demográfico, socioeconómico y laboral del mercado, el sistema de regulación laboral, y las características del sistema de educación, tienen mayor incidencia en este proceso.

Al respecto, Muñoz (2006) expone que, en los países con sistemas educativos de calidad poco demostrada, frágiles vínculos con el mercado de trabajo y en los que el proceso de transición de formación profesional al trabajo experimenta mayores restricciones, son los que presentan tasas de desempleo juvenil muy altas en comparación con las de las personas adultas. Por su parte, Morales (2016) señala que la población juvenil afronta barreras en su inserción laboral. El déficit de trabajo decente en este grupo de la población es significativo y heterogéneo, lo que implica bajos ingresos, inestabilidad y desprotección social.

Para la OIT (2018) el desajuste
entre las cualificaciones para desempeñar puestos de trabajo y las que poseen las personas que lo solicitan, y la falta de correspondencia entre la oferta y la demanda de trabajo por poca transparencia en los mercados laborales no permite una pronta $y$ oportuna orientación vocacional. Esto implica vacíos en la oferta de los sistemas educativos, lo que deriva a una mayor tasa de desempleo y a una 
Villalobos Rodríguez, Gerardo; Moraga López, Geannina;

Guevara Portuguez, María José y Araya-Castillo, Luis

Desempleo juvenil: contribuciones para su disminución desde el emprendimiento

profundización de las limitaciones y barreras de entrada al mundo del trabajo.

Posteriormente, la OIT (2019) plantea que estas limitaciones son producto del desconocimiento de las características de la demanda del mercado, de los rápidos cambios tecnológicos en que se encuentra sumergida la economía, y de la tendencia de las empresas a elevar sus requisitos en relación con las calificaciones de la fuerza laboral.

Por otra parte, De La Hoz, Quejada \& Yánez (2012), constatan la relación entre el desempleo juvenil y la dinámica de la actividad económica. Demidova y Signorelli (2010), y Choudhry, Marelli \& Signorelli (2010) analizan que un insuficiente crecimiento económico acrecienta el desempleo en general y el de los jóvenes en particular, lo que favorece la vulnerabilidad al riesgo de inactividad prolongada y exclusión social.

Para Lépore y Schleser (s.f.), en periodos de recesión económica, los hogares acostumbran a extender su oferta laboral, mediante el aporte de los miembros más jóvenes en los mercados de trabajo. Además, las empresas dejan de contratar personal, y generalmente suelen ser los jóvenes los primeros en ser despedidos debido a su baja productividad o a los mayores costos que implican los despidos del personal con mayor antigüedad y con más experiencia.

La OCDE (2017) evidencia que las personas jóvenes son más vulnerables a las crisis económicas; por ejemplo, entre los años 2008 y 2009 , en la crisis económica que afectó gran parte del mundo, el número de jóvenes desempleados creció en un punto porcentual, mientras que la de las personas adultas se incrementó en $0,5 \%$.
Los ingresos familiares, el estrato económico, el entorno familiar y las características ocupacionales de los padres y madres son aspectos que también inciden en la situación laboral de los jóvenes (De La Hoz et al., 2012). Dentro del círculo de la familia los miembros mayores y con conexiones pueden recomendar al joven para un puesto de trabajo, y esto indudablemente le facilita la posibilidad de colocarse en el mercado laboral.

La situación laboral y nivel educativo de los padres y madres también incide en la situación laboral de los jóvenes. En el caso de los hogares donde el padre y la madre presentan altos niveles de escolaridad y trabajos estables, a los hijos se les facilita más la incorporación a un empleo con mejores condiciones en cuanto a la formalidad, estabilidad y posibilidades de desarrollo profesional (De La Hoz et al., 2012).

Para Loría y Salas (2019) los jóvenes con menor educación y provenientes de familias con ingresos familiares más bajos, enfrentan una mayor tasa de desempleo que los jóvenes con mayor educación y de familias con ingresos altos. Además, el primer grupo tiende a ocuparse en empleos de baja remuneración y de alta precariedad, representando una heterogeneidad importante en esta población.

Por otro lado, Borda (2015) indica que la incorporación de los jóvenes a la actividad económica requiere no solo del dinamismo del sector privado para crear puestos de trabajo, sino también de políticas públicas ágiles y efectivas que aprovechen el activo demográfico de los países para promover más crecimiento, mayor bienestar y menores niveles de pobreza.

Siguiendo con esa perspectiva, desde el punto de vista emocional de la 
población joven, la falta de oportunidades laborales puede transformarse en un problema asociado a trastornos psicológicos, que se refleja en desánimo y muchas veces los empuja a los atajos fáciles para resolver sus necesidades, con el riesgo de convertirlos en factores de inestabilidad social. Borda (2015) indica que estos efectos implican consecuencias irreversibles en las perspectivas futuras de empleo, en la formación de capital humano y en la capacidad de integrarse al mercado de trabajo.

Otros análisis relacionados con lo expuesto, es que las personas que experimentan periodos de desempleo a edades tempranas presentan una mayor probabilidad de tener periodos de desempleo en el futuro y restricciones en la generación de ingreso (Weller, 2007).

\section{Desempleo juvenil en Latinoamérica: evidencias en época pre- pandemia de la COVID-19}

Con el objetivo de mostrar la dimensión del desempleo juvenil en América Latina, la OIT confirma que para el año 2019 y después de haber mostrado una reducción por varios años, la tasa de desempleo mundial de los jóvenes ha aumentado. Además, para los jóvenes que trabajan principalmente en el sector informal, el pronóstico es poco favorable para poner fin a la pobreza de aquí al 2030 , si no se redoblan los esfuerzos para alcanzar un crecimiento económico sostenible.

Previo a esas conclusiones, en el año 2018 la OIT advierte sobre la problemática que representa el desempleo juvenil y cuantifica lo siguiente:

- Entre los años 1998 y 2018, la población joven aumentó en 139 millones de personas, mientras que la fuerza de trabajo juvenil disminuyó en 35 millones de personas. Esta dinámica también está reflejada en una proporción decreciente de jóvenes en la fuerza de trabajo mundial, la cual pasó del $21,7 \%$ en el año 1997 al $15,5 \%$ en el año 2018.

- $\quad$ En 2018 el 16,7\% de los jóvenes que trabajan en las economías emergentes y en desarrollo viven por debajo del umbral de extrema pobreza de 1,90 dólares americanos por día.

- Entre la actualidad y el año 2030 se estima que 25,6 millones de jóvenes trabajadores entre los 15 y 29 años ingresarán a la fuerza de trabajo y necesitarán empleos.

Para la OIT (2020c) las principales cifras sobre la problemática del desempleo juvenil en la región latinoamericana se muestran en el gráfico 1, lo que evidencia que algunos países como Costa Rica, Brasil y Argentina presentan las tasas más altas de desempleo juvenil. En Costa Rica particularmente el desempleo juvenil aumentó en más de diez puntos porcentuales del 2019 al 2020, pasando de un $31.1 \%$ a un $42,1 \%$. Por otro lado, en los casos de México, Ecuador y Perú, el desempleo juvenil resulta mucho más bajo. Sin embargo, en estos países las tasas de empleo juvenil informal son muy altas, según el mismo reporte. 


\section{Gráfico 1 \\ América Latina y el Caribe: tasa de desempleo juvenil por país, en porcentajes, años 2019 y 2020}

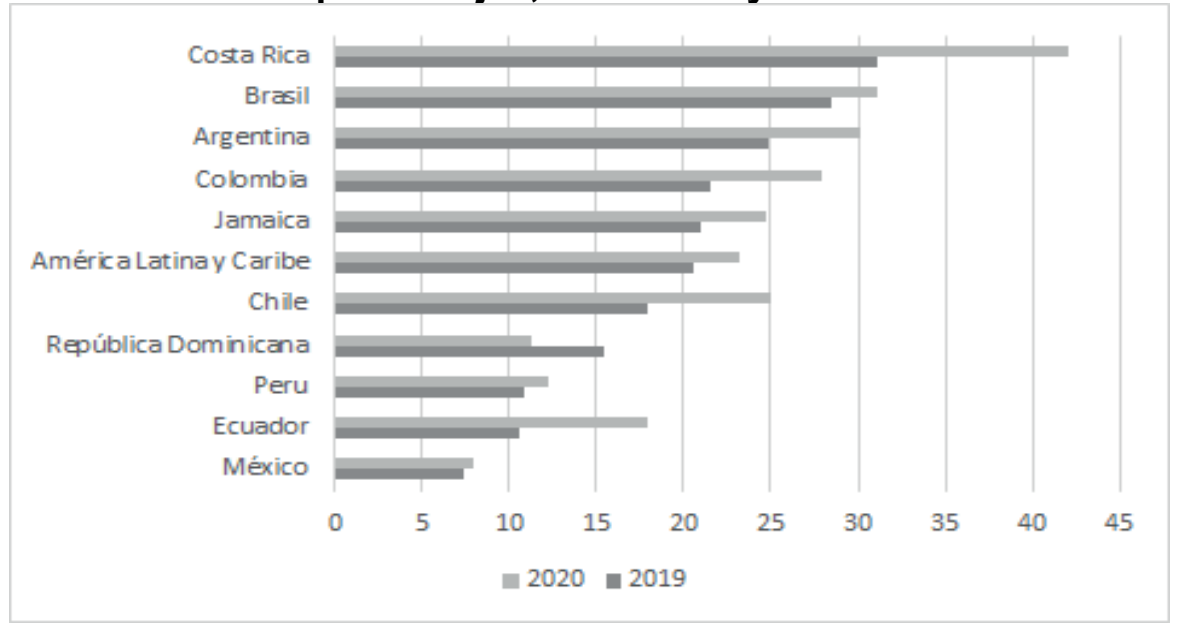

Fuente: Las encuestas rápidas para captar los efectos sobre la ocupación y el trabajo no remunerado (OIT, 2020c).

Recientemente, la OIT (2020a) confirma que previo a la crisis sanitaria por la COVID-19 en América Latina y el Caribe, la situación laboral de los jóvenes mostraba enormes desafíos para los gobiernos de estos países, pues se trata de un segmento de la población asediada por altos niveles de desempleo e informalidad.

La OIT muestra como para el año 2019 se registra en la región 107,9 millones de jóvenes entre 15 y 24 años, de los cuales 52,7 millones formaban parte de la fuerza de trabajo. Los otros 55,2 millones de jóvenes continuaban en actividades de educación exclusivamente, o estaban en situación de inactividad. Del porcentaje que formaba parte de la fuerza de trabajo, a fines de 2019 la tasa de desocupación juvenil llegó a $17,9 \%$, lo que implicaba que unos 9,4 millones de jóvenes se encontraban en situación de desempleo. Esto significa casi el $40 \%$ del total de desempleados en la región.

Además, la OIT (2019) evidencia que en la región la informalidad afectaba a alrededor del $60 \%$ de los trabajadores jóvenes, lo cual significa que 6 de cada 10 jóvenes que sí lograban insertarse en los mercados laborales solamente encontraban ocupación en condiciones de informalidad. Esta situación agrava las condiciones de vulnerabilidad ante la pérdida de empleos y la pérdida de ingresos en medio de una crisis sanitaria como la iniciada en el año 2020 por el COVID-19.

Adicional a lo anterior, la OIT (2021) advierte que después de la pandemia la informalidad laboral juvenil se incrementó de forma notable. Por ejemplo, el déficit de trabajo formal se hará más evidente para ciertos grupos de trabajadores 
como los jóvenes, las mujeres y los adultos con menores calificaciones, grupos que estructuralmente exhiben mayores dificultades para insertarse en un puesto formal.

\section{Efectos de la COVID-19 en el empleo de los jóvenes latinoamericanos}

El desempleo juvenil es un problema social que se ha exacerbado tras la pandemia del COVID-19 (Dávila, Zlobina y Pascual, 2021). Un estudio reciente de la CEPAL (2020) indica que el desempeño económico y laboral de América Latina y el Caribe se encontraba en una situación de estancamiento desde el año 2019, cuando la tasa de desocupación alcanzó un $8 \%$ y afectó aproximadamente a 25,7 millones de personas. La paralización de las actividades productivas y laborales, como parte de las medidas sanitarias para combatir la propagación del virus SARS-Cov-2 impactó fuertemente a los mercados laborales de todos los países de la región.

Tomado del mismo estudio de la CEPAL (2020), algunas actividades laborales se han podido mantener a través del trabajo remoto $y$ el teletrabajo, sin embargo, corresponde a una proporción relativamente baja de trabajadores en la región, en promedio solo el $23 \%$ de los ocupados puede mantener una modalidad de teletrabajo o trabajo a distancia.

Países como Costa Rica, Colombia, México y Chile, han implementado medidas para proteger el empleo, entre las que se encuentran las licencias anticipadas o la reducción de jornadas laborales o salario, lo cual trae consecuencias directas, como por ejemplo un salario reducido en detrimento de las personas trabajadoras (OIT, 2021).

En cuanto a evidencia reportada, los sectores e industrias más afectadas por la pandemia en la región, la OIT (2020d) registra caídas drásticas del empleo en el sector servicios, destacando actividades asociadas al turismo como el alojamiento y el servicio de comidas, el comercio, la construcción y la manufactura. También significativas disminuciones del empleo en las actividades artísticas y de entretenimiento.

En contraposición, los sectores en que se registra un aumento de empleo se asocian a la administración pública o al suministro de servicios básicos esenciales, como agua y electricidad. Muchas de las actividades que han sufrido una disminución drástica del empleo, históricamente han sido fuentes laborales seguras para las poblaciones más jóvenes, como por ejemplo el caso del turismo, entretenimiento y el comercio.

Relacionado con lo anterior, la OIT (2020c) ha estimado que a nivel mundial 4 de cada 10 jóvenes previo a la pandemia, se desempeñaban en los sectores que han resultado más fuertemente afectados, como el turismo, el comercio y los servicios en general. Otro factor que ha golpeado a los jóvenes latinoamericanos es la pérdida de ingresos de los trabajadores en condiciones de informalidad, dado que alrededor del $60 \%$ de los ocupados jóvenes se desempeñan en esas condiciones.

La OIT (2020d) presentó estimaciones según las cuales el $80 \%$ de los trabajadores informales de la región están afectados por una severa caída en sus ingresos, junto a un aumento abrupto de la pobreza que afectaría a 
Villalobos Rodríguez, Gerardo; Moraga López, Geannina;

Guevara Portuguez, María José y Araya-Castillo, Luis

Desempleo juvenil: contribuciones para su disminución desde el emprendimiento

cerca del $90 \%$ de las personas ocupadas en condiciones de informalidad.

Como alternativas, la OIT (2020d) presentó medidas ante la crisis de la COVID-19 para contrarrestar los efectos en el mercado laboral de los jóvenes de la región latinoamericana. Entre las principales se mencionan:

- Centrarse en la formación. El fortalecimiento de las capacidades profesionales de los jóvenes con orientación hacia la formación emprendedora, principalmente en las universidades.

- Apoyar a los jóvenes emprendedores. El trabajo por cuenta propia es una plataforma para que los jóvenes obtengan independencia económica.

- Apoyar mediante políticas concretas la conversión de emprendimientos tradicionales y por necesidad a emprendimientos por oportunidad, intensivos en conocimiento $y$ más dinámicos, para favorecer la empleabilidad.

Por otro lado, previo a la pandemia por la COVID-19 se consideraba la digitalización de ciertos servicios como un factor clave para la competitividad, porque no sólo permite una comunicación más fluida, sino también el acceso a un mercado abierto e impulsa el desarrollo de nuevos procesos, productos y servicios (Martínez y Rubio, 2020).

Adicional, considerandoloexpuesto por Marí y Cervantes (2020) quienes mencionan que la sociedad se ha vuelto más exigente, no solo en la evaluación del comportamiento de las empresas en relación con su gestión y formas de gobernanza, sino también en el impacto en el entorno social y medioambiental. Es decir, los consumidores en general están prefiriendo productos y servicios que evidencien un impacto favorable en temas sociales y ambientales (proyectos de triple impacto).

Estos planteamientos no sólo se abordan como recomendaciones para los jóvenes emprendedores, sino que también confiere una responsabilidad a las instituciones de educación superior para que contribuyan con el fortalecimiento de las capacidades y a los gobiernos o ministerios para que fomenten políticas específicas a este grupo de la población.

\section{Emprendimiento y nuevas empresas para la generación de empleos}

El emprendimiento se ha entendido como un impulsor de nuevo empleo, que aporta al bienestar económico, por lo cual muchos programas de política pública se han enfocado en la creación y fortalecimiento de estos (Rodríguez y Rehner, 2021). Diversos organismos, como la OIT, la OCDE, la CEPAL y el Banco Mundial caracterizan a los nuevos emprendimientos como una de las herramientas más efectivas en la generación de nuevos empleos.

Para la OCDE (2018) una de las competencias que se debe fomentar en los jóvenes latinoamericanos es el emprendimiento como vía principal para mejorar la empleabilidad y la movilidad social. Los jóvenes de la región demuestran gran capacidad creativa, de gestión de grupos y proyectos, de toma de riesgos y de trabajo en busca de objetivos concretos.

Por otro lado, el Organismo Internacional de Juventud (2018) asegura que América Latina muestra predisposición favorable hacia el emprendimiento, ya que casi el $72,6 \%$ 
de jóvenes entre 18-24 años perciben el emprendimiento como una buena elección de desarrollo profesional, y el $69,51 \%$ cree que los emprendedores gozan de una alta consideración social.

Autores como Alarcón y Ochoa (2021) destacan que el emprendimiento visto como resultado de los procesos de creatividad e innovación, se hace cada vez más común en la sociedad contemporánea, y constituye una de las estrategias para la incorporación del ser humano en los procesos productivos. Desde esta perspectiva, sin distinción etaria, la ruta al emprendimiento no solo favorece el desarrollo social y económico de un territorio, sino el campo profesional de las personas.

El impulso a las iniciativas emprendedoras ha demostrado ser una opción eficiente que contribuye a la generación de nuevos empleos tanto en economías desarrolladas como en vías de desarrollo. Por ejemplo, para la OIT (2016) existen sólidos datos empíricos que confirman que los emprendimientos y las pequeñas y medianas empresas (PYME) son un verdadero motor de creación de empleo. Las PYME de reciente creación son las que más contribuyen a la creación de empleo en la región latinoamericana.

Relacionado con lo anterior, la
OIT (2016) evidencia que las empresas de reciente creación son agentes económicos clave para la promover el desarrollo de los países de América Latina y el Caribe. Un alto porcentaje de la población (incluyendo los jóvenes) depende de la actividad y el desempeño de las nuevas empresas debido a su capacidad para la generación de empleo y su participación en la producción.

Un argumento similar, dado por Kantis (2014), quien argumenta sobre la importancia de los emprendimientos en la economía, ya que representan más del $90 \%$ del total de empresas en la mayoría de los países del mundo, generan un alto volumen de empleo y contribuyen al desarrollo económico.

Algunas cifras de la CEPAL (2020b), tal y como se muestra en el gráfico 2, respecto a la contribución en el empleo en América Latina, reflejan que entre las micro y pequeñas empresas contribuyen con el $47,1 \%$ de los empleos del sector privado de la región, en contraposición las empresas grandes aportan el 38,8\% del empleo, mientras que las medianas el $14 \%$. Resulta concluyente que los emprendimientos, empresas de reciente creación y micro y pequeñas empresas están contribuyendo con casi el $50 \%$ del empleo en América Latina. 


\section{Gráfico 2 \\ Contribución del empleo privado según tamaño de empresa. Latinoamérica, 2020}

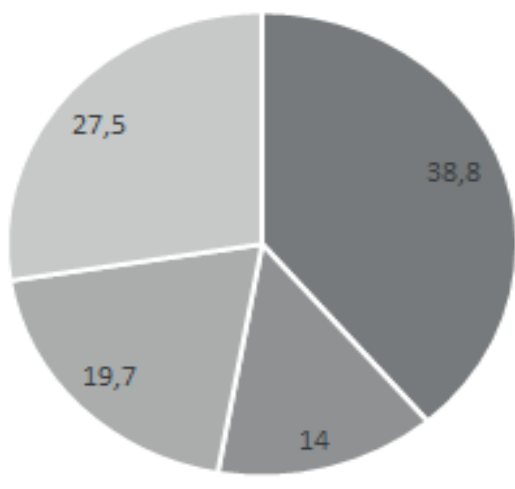

- Grandes empresas |- Medianas empresas || Pequeñas empresas | Microempr esas

Fuente: Elaboración propia, a partir de los datos de CEPAL (2020).

En seguimiento a lo planteado y según Amorós (2011), el Global Entreprenuership Monitor (GEM) es el proyecto de investigación más importante a escala mundial que mide el emprendimiento y los factores que inciden en el aumento o disminución de la tasa de actividad emprendedora (TAE).

Tomado del mismo autor, la tasa de actividad emprendedora muestra el porcentaje de la población que está en el camino de iniciar o que recientemente puso en marcha un emprendimiento. Según el GEM (2019), la juventud mayor (25 a 34 años) es el grupo más emprendedor en todos los países latinoamericanos, mostrando que el índice de empresas consolidadas para la juventud mayor es 2,4 veces mayor que para la juventud joven (18 a 24 años). La baja tasa de empresa consolidada entre la juventud joven es una preocupación menor, ya que es probable que un porcentaje importante de individuos de este grupo estén participado en procesos de educación secundaria y universitaria.

Relacionado con lo anterior, para Peña, Garcés \& Serafim (2020), el grado de innovación e intención emprendedora están asociados con la autoeficacia y educación del emprendedor; donde la población más exitosa han sido emprendedores universitarios. Por esta razón, y con base en Terán, Valdez \& Miranda (2020), en una sociedad basada en el conocimiento, la universidad juega un rol transformador del conocimiento con el escalamiento de este en una iniciativa emprendedora. 
En un estudio reciente, realizado por Martínez, Durán \& Serna (2021) que analiza las intenciones emprendedoras de 152 estudiantes universitarios en el contexto actual de pandemia por la COVID-19, se destaca que el emprendimiento es una alternativa sostenible para la empleabilidad, los ingresos y el bienestar de esta población.

A partir de lo expuesto, se considera necesario establecer diferencias entre el emprendimiento por necesidad y por oportunidad. Para Amóros y Poblete (2013) el emprendimiento por necesidad o subsistencia es aquel que surge por una necesidad puntual de la persona emprendedora, principalmente de tipo económico, tiene poca innovación, se asocia a personas de escolaridad baja, tiene pocas posibilidades de crecimiento y su impacto económico y social es bajo.
Por otra parte, el emprendimiento por oportunidad se asocia a la identificación de oportunidades de mercado, incorporan la innovación, son intensivos en conocimiento, se asocian a personas de mayor escolaridad y su impacto positivo en la sociedad es mayor (Villalobos et al., 2017). Por las razones anteriores, organismos como la OCDE (2017) recomiendan políticas y programas orientados a potenciar el emprendimiento por oportunidad.

Aunado a lo anterior, el GEM (2018) revela que antes de la pandemia COVID-19, los jóvenes latinoamericanos mostraban altos niveles de motivación para crear emprendimientos por oportunidad, ya que cerca de tres cuartas partes de los jóvenes emprendedores están motivados por la oportunidad. 
Villalobos Rodríguez, Gerardo; Moraga López, Geannina;

Guevara Portuguez, María José y Araya-Castillo, Luis

Desempleo juvenil: contribuciones para su disminución desde el emprendimiento

\begin{tabular}{|c|c|c|c|c|c|c|}
\hline \multirow[b]{2}{*}{ País } & \multicolumn{3}{|c|}{$18-24$ años } & \multicolumn{3}{|c|}{$25-34$ años } \\
\hline & $\begin{array}{l}\text { Motivados por } \\
\text { la oportunidad }\end{array}$ & $\begin{array}{l}\text { Motivados } \\
\text { por la } \\
\text { necesidad }\end{array}$ & $\begin{array}{l}\text { Proporción } \\
\text { oportunidad/ } \\
\text { necesidad }\end{array}$ & $\begin{array}{l}\text { Motivados } \\
\text { por la } \\
\text { oportunidad }\end{array}$ & $\begin{array}{l}\text { Motivados } \\
\text { por la } \\
\text { necesidad }\end{array}$ & $\begin{array}{l}\text { Proporción } \\
\text { oportunidad/ } \\
\text { necesidad }\end{array}$ \\
\hline Argentina & 69,4 & 27,2 & 2,5 & 59,7 & 37,0 & 1,6 \\
\hline Bolivia & 81,6 & 18,4 & 4,4 & 80,0 & 19,0 & 4,2 \\
\hline Brasil & 53,9 & 46,1 & 1,2 & 63,6 & 36,4 & 1,7 \\
\hline Chile & 82,7 & 14,9 & 5,6 & 71,4 & 21,7 & 3,3 \\
\hline Colombia & 70,7 & 24,2 & 2,9 & 69,0 & 31,0 & 2,2 \\
\hline Costa Rica & 86,9 & 11,9 & 7,3 & 80,7 & 18,8 & 4,3 \\
\hline Guatemala & 53,4 & 44,6 & 1,2 & 60,1 & 39,9 & 1,5 \\
\hline México & 76,3 & 21,1 & 3,6 & 79,3 & 18,8 & 4,2 \\
\hline Panamá & 78,4 & 21,6 & 3,6 & 50,0 & 47,2 & 1,1 \\
\hline Perú & 70,7 & 25,3 & 2,8 & 75,4 & 24,6 & 3,1 \\
\hline Uruguay & 83,0 & 17,0 & 4,9 & 83,3 & 14,9 & 5,6 \\
\hline
\end{tabular}

Fuente: Elaboración propia, a partir de datos del GEM (2018)

Países como Bolivia, Chile, Costa Rica y Uruguay destacan en los dos grupos etarios (18-24 años y 25-34 años) donde se refleja una mayor motivación para emprender por oportunidad y no por necesidad. En estos casos la proporción oportunidad/necesidad es mayor a 4,5. Caso contrario sucede en países como Brasil, Guatemala y Panamá, donde la evidencia muestra una proporción necesidad/oportunidad por debajo de 2 en ambos grupos etarios.

Otro indicador relacionado con la posibilidad de emprender es el número de empleos creados. De acuerdo con el GEM (2018), en promedio, un tercio de los jóvenes emprendedores iberoamericanos solo crean empleo para el propietario de la empresa (autoempleo) y, por lo tanto, no contribuye de forma significativa a la creación de empleo. Sin embargo, para muchas economías, pero principalmente las emergentes, cada empleo cuenta, y no es inusual identificar un trabajador por cuenta propia que mantenga a una familia y asegure el mejoramiento de su calidad de vida. 


\section{Tabla 2}

\section{Creación de empleo real por empresas jóvenes en la fase inicial en países latinoamericanos, 2017}

\begin{tabular}{|c|c|c|c|c|c|c|}
\hline \multirow[b]{2}{*}{ País } & \multicolumn{3}{|c|}{$18-24$ años } & \multicolumn{3}{|c|}{$25-34$ años } \\
\hline & $\begin{array}{c}\text { Cero } \\
\text { empleos }\end{array}$ & 1-5 empleos & $\begin{array}{l}6 \text { o más } \\
\text { empleos }\end{array}$ & $\begin{array}{c}\text { Cero } \\
\text { empleos }\end{array}$ & 1-5 empleos & $\begin{array}{l}6 \text { o más } \\
\text { empleos }\end{array}$ \\
\hline Argentina & 29,9 & 51,1 & 19,0 & 37,6 & 47,1 & 15,3 \\
\hline Bolivia & 46,2 & 50,0 & 3,7 & 24,7 & 71,3 & 4,0 \\
\hline Brasil & 62,6 & 32,7 & 4,8 & 54,4 & 37,7 & 7,9 \\
\hline Chile & 10,6 & 61,6 & 27,8 & 19,3 & 45,3 & 35,3 \\
\hline Colombia & 11,7 & 26,6 & 61,7 & 14,2 & 31,3 & 54,4 \\
\hline Costa Rica & 40,7 & 55,6 & 3,7 & 48,1 & 42,3 & 9,6 \\
\hline Guatemala & 13,0 & 73,3 & 13,8 & 18,8 & 70,9 & 10.3 \\
\hline México & 42,2 & 45,2 & 12,6 & 52,5 & 38,2 & 9,3 \\
\hline Panamá & 54,1 & 45,9 & 0,0 & 45,8 & 52,8 & 1,4 \\
\hline Perú & 24,1 & 56,6 & 19,2 & 37,0 & 49,4 & 13,6 \\
\hline Uruguay & 33,2 & 31,6 & 35,3 & 38,9 & 45,0 & 16,1 \\
\hline
\end{tabular}

Fuente: Elaboración propia, según GEM (2018)

\section{El estudio del GEM (2018)} revela que algunos de los países latinoamericanos destacan en el tema de creación de empleo. Por ejemplo, para el caso de Colombia, casi un $62 \%$ de los emprendedores entre 18 y 24 años están contratando más de seis empleados, y en el rango de edad entre 25 y 34 años, el porcentaje llega casi al 55\%.

Otro país que sobresale en este estudio es Chile, donde el $28 \%$ de los emprendedores entre 18 y 24 años generan más de 6 empleos, y en el rango de 25-34 años el porcentaje es de $35 \%$. Caso contrario sucede en países como Panamá, Bolivia y Costa Rica, donde los jóvenes emprendedores se encuentran muy por debajo de la media latinoamericana en ambos estratos de edad en cuanto a la generación de seis o más empleos.

\section{Conclusiones}

El desempleo en la población joven es un problema generalizado que afecta a los países de la región latinoamericana, lo cual se asentó con la pandemia del COVID-19, la cual no solamente provocó una gran pérdida de empleos en todos los países de la región, sino que contribuyó a potenciar otras problemáticas sociales y económicas como la pobreza.

El estudio permitió identificar algunas de las causas que detonan el desempleo juvenil, siendo las principales los bajos niveles de escolaridad, patrones familiares, los altos índices demográficos que experimentan algunos países, y el desequilibrio entre oferta y demanda con respecto al grado de cualificaciones para desempeñar algunos puestos de trabajo 
Villalobos Rodríguez, Gerardo; Moraga López, Geannina;

Guevara Portuguez, María José y Araya-Castillo, Luis

Desempleo juvenil: contribuciones para su disminución desde el emprendimiento

con requisitos específicos.

Además, se pone de manifiesto que las consecuencias derivadas por la falta de oportunidades laborales, favorece la probabilidad de caer bajo la línea de la pobreza, el incremento de los trastornos psicológicos causados por el desánimo y la desmotivación de los jóvenes, la migración, la búsqueda de soluciones fáciles para resolver sus necesidades, con el riesgo de caer en la delincuencia, el narcotráfico y la prostitución y otros factores de inestabilidad social y la pérdida de capital humano que tengan las competencias necesarias para integrarse al mercado de trabajo.

Se constata que el emprendimiento es una herramienta clave para atender directamente la problemática del desempleo, especialmente los de tipo "por oportunidad" porque contribuyen con la generación de nuevos puestos de trabajo en la mayoría de los países latinoamericanos.

La juventud latinoamericana muestra un interés creciente a emprender. La tasa de actividad emprendedora (TAE) de los países de la región muestra expectativa favorable, especialmente para la población joven entre los 25 a los 34 años y cuando son motivados por una oportunidad.

Los emprendimientos creados por personas jóvenes están generando fuentes de empleo en los países latinoamericanos. No solamente a través del autoempleo, sino que un porcentaje significativo de los nuevos emprendimientos brindan más de seis empleos, siendo relevantes en este caso países como Colombia, Chile y Uruguay.

Las evidencias mostradas en este artículo conducen a reflexionar sobre la relevancia y urgencia de que los países latinoamericanos desarrollen políticas claras que permitan generar condiciones sistémicas favorables para el impulso al emprendimiento, principalmente en las personas jóvenes.

Las nuevas generaciones muestran gran interés por emprender y por ser parte de la solución, brindando nuevas fuentes de empleo; y no parte del problema siendo un número más en las estadísticas de desempleo.

\section{Referencias bibliográficas}

\section{Alarcón U., \& Ochoa, A. (2021).} Entrepreneurship as a collective practice: A case study in the lake region. [Emprendimiento como práctica colectiva en la región de los lagos]. Revista Venezolana De Gerencia, 26(94), 802-817. https:// doi.org/10.52080/rvgluzv26n94.19

Amorós, J. (2011). El proyecto Global Entrepreneurship Monitor (GEM): una aproximación desde el contexto latinoamericano. Revista Latinoamericana de Administración, 46, 1-15. https://www.redalyc.org/ articulo.oa?id=71617238002

Amorós, J., \&amp; Poblete, C. (2013). Aspiraciones de los emprendedores en Chile y el mundo 2012. Global Entrepreneurship Monitor. https:// repositorio.udd.cl/bitstream/ handle/11447/670/GEM-Chile-2012Reporte-Aspiracion-Emprendedora. pdf? sequence=1\&amp;isAllowed=y

Banco Mundial (2019). Informe anual 2019 Poner fin a la pobreza, invertir para generar oportunidades. https:// documents1.worldbank.org/curated/ en/328341570462983234/pdf/ The-World-Bank-Annual-Report2019-Ending-Poverty-Investing-inOpportunity.pdf

Borda, D. (2015). Inserción de los jóvenes en el mercado de trabajo. Centro Internacional de Investigación para el Desarrollo. https:// 
biblioteca.clacso.edu.ar/Paraguay/ cadep/20160714115802/10.pdf

Brunner, J., Ganga, F., \& Labraña, J. (2020). Universidad y protesta social: una reflexión desde Chile. Revista Iberoamericana de Educación Superior, 11(32), 3-22. $\quad$ https://doi.org/10.22201/ iisue.20072872e.2020.32.814

Choudhry, M., Marelli, E., \& Signorelli, M. (2010). The impact of financial crises on youth unemployment rate. Quaderni del Dipartimento di Economia, Finanza e Statistica, 79, 1-18. https://econpapers.repec.org/ paper/piawpaper/79 2f2010.htm

Comisión Económica paraAmérica Latina y el Caribe- CEPAL (2020b). Sectores y empresas frente al COVID-19: emergencia y reactivación. Informe especial. https://repositorio.cepal. org/bitstream/handle/11362/45734/4/ $\underline{\text { S2000438 es.pdf }}$

Comisión Económica paraAmérica Latina y el Caribe- CEPAL. (2020a). Estudio Económico de América Latina y el Caribe, 2020 (LC/PUB.2020/12-P), Santiago, 2020 https://www.cepal. org/sites/default/files/publication/ files/46070/S2000371_es.pdf

Dávila, M. C., Zlobina, A., \& Pascual, A. S. (2021). Emprendimiento social en jóvenes: Análisis de su relación con otras formas de participación social REVESCO. Revista De Estudios Cooperativos, 138, 1-14. https://doi. org/10.5209/REVE.75562

De La Hoz, F., Quejada, R., \& Yánez, M. (2012). El desempleo juvenil: problema de efectos perpetuos. Revista Latinoamericana de Ciencias Sociales, Niñez y Juventud, 10(1), 427-439. https://www.scielo.org.co/ pdf/rlcs/v10n1/v10n1a27.pdf

Demidova, O., \& Signorelli, M. (2010). The impact of crises on youth unemployment of Russian Regions: An empirical analysis. Quaderni del Dipartimento di Economia, Finanza e Statistica, 41. https://core.ac.uk/ download/pdf/6244826.pdf

Global Entrepreneurship Monitor (2018). 2017 United States Report. Babson College. https://www.babson. edu/media/babson/site-assets/ content-assets/academics/centersand-institutes/the-arthur-m-blankcenter-for-entrepreneurship/globalresearch/GEM USA 2017.pdf

Global Entrepreneurship Monitor (2019). 2018/2019 Global Report. Babson College. https://www. babson.edu/media/babson/siteassets/content-assets/academics/ centers-and-institutes/the-arthur-mblank-center-for-entrepreneurship/ global-research/gem-2018-2019global-report.pdf

Global Entrepreneurship Monitor (2020). 2019/2020 Global Report. Babson College. https://www.gemconsortium. org/file/open?fileld $=50443$

Global Entrepreneurship Monitor (2021). 2020/2021 Global Report. Babson College. https://www.gemconsortium. org/file/open?fileld=50691

Kantis, H., Federico, J., \& Ibarra, S. (2014). Índice de condiciones sistémicas para el emprendimiento dinámico: una herramienta para la acción en América Latina. (1a ed.). Rafaela: Asociación Civil Red Pymes Mercosur. https:// prodem.ungs.edu.ar/wp-content/ uploads $/ 2018 / 03 / \%$ C3\%8Dndicede-condiciones-sist $\%$ C3\%A9micaspara-el-emprendimientodin\%C3\%A1mico.-Una-herramientapara-la-acci\%C3\%B3n-enAm\%C3\%A9rica-Latina.pdf

Klisberg, B. (2007). Hacia una Economía con rostro Humano. Cuadernos Latinoamericanos de Administración, 
Villalobos Rodríguez, Gerardo; Moraga López, Geannina;

Guevara Portuguez, María José y Araya-Castillo, Luis

Desempleo juvenil: contribuciones para su disminución desde el emprendimiento

1(I), 117. https://www.redalyc.org/ articulo.oa? id=409634371016

Lépore, E., \& Schleser, D. (s.f.). Diagnóstico del desempleo juvenil. Subsecretaría de Programación Técnica y Estudios Laborales. Argentina. https://www.trabajo. gob.ar/downloads/biblioteca estadisticas/toe2 07diagnosticodesempleo.pdf

Loría, E., \& Salas, E. G. (2019). ¿El Desempleo Juvenil en México es Voluntario? Estudios de Economía Aplicada, 37(2), 1-14. https://dialnet.unirioja.es/servlet/ articulo?codigo $=6963184$

Marí, P. A., \& Cervantes, C. R. (2020). Presentación monográfico: La economía social ante sus retos: Emprendimiento, transformación digital, género y desarrollo sostenible. Revista De Estudios Cooperativos, $133 . \quad$ https://doi.org/10.5209/ reve. 69000

Martínez Garcés, J., Durán Omaña, S., \& Serna Borja, W. (2021). COVID-19, educación en emprendimiento e intenciones de emprender: Factores decisorios en estudiantes universitarios. Revista De Ciencias Sociales, 27(2), 272-283. https://doi. org/10.31876/rcs.v27i2.35913

Martínez, N., \& Bañón, R. (2020). Emprendimiento en épocas de crisis: Un análisis exploratorio de los efectos de la COVID-19. Small Business International Review, 4(2), 53-66. https://doi.org/10.26784/sbir. $\underline{\mathrm{v} 4 \mathrm{i} 2.279}$

Morales, M. A. (2016). Estrategias para atender el empleo juvenil en la economía informal. Revista Latinoamericana de Derecho Social, 23, 89-121. https://doi.org/10.1016/j. rlds.2016.04.002

Muñoz, C. (2006). Determinantes de la empleabilidad de los jóvenes universitarios y alternativas para promoverla. Papeles de Población, 49, 75-89. https://bit.ly/3r9VFfX

OIT (2021). Perspectivas sociales y del empleo en el mundo: tendencias 2021. Informe. https://www.ilo.org/ wcmsp5/groups/public/---dgreports/--dcomm/---publ/documents/ publication/wcms 794492.pdf

Organismo Internacional de Juventud (2018). Emprendimiento Juvenil en Iberoamérica: una clave para la empleabilidad. https://www. segib.org/wp-content/uploads/ INFORME-EMPRENDIMIENTOIBEROAMERICA-OIJ.pdf

Organización Internacional del TrabajoOIT (2016). Trabajo Decente y la Agenda 2030 de Desarrollo Sostenible. Estado de Trabajo Decente en el Mundo. https://www. ilo.org/global/topics/sdg-2030/lang-es/index.htm

Organización Internacional del TrabajoOIT (2018). Panorama Laboral 2018: América Latina y el Caribe. Lima: Oficina Regional para América Latina y el Caribe. https://www.ilo.org/ wcmsp5/groups/public/---americas/-ro-lima/documents/publication/ wcms 654969.pdf

Organización Internacional del TrabajoOIT (2019). Nota de política: Un enfoque sistémico para crear más y mejores Empleos. Ginebra, Suiza. https://www.ilo.org/wcmsp5/groups/ public/---ed emp/---emp ent/--ifp seed/documents/publication/ wcms 732834.pdf

Organización Internacional del TrabajoOIT (2020a). Empleo juvenil en tiempos de la COVID-19: el riesgo de una generación del confinamiento. Nota conceptual. https://www.ilo.org/ wcmsp5/groups/public/---americas/--ro-lima/documents/briefingnote/ 


\section{wcms 753103.pdf}

Organización Internacional del TrabajoOIT (2020b). Panorama laboral en tiempos de la COVID-19: impactos en el mercado de trabajo y los ingresos en América Latina y el Caribe. Nota técnica. https://www.ilo.org/ wcmsp5/groups/public/---americas/--ro-lima/documents/publication/ wcms 749659.pdf

Organización Internacional del TrabajoOIT (2020c). Las encuestas rápidas para captar los efectos sobre la ocupación y el trabajo no remunerado. Nota técnica. https:// www.ilo.org/wcmsp5/groups/public/--dgreports/---stat/documents/ publication/wcms 750215.pdf

Organización Internacional del TrabajoOIT (2020d). Observatorio de la OIT: El COVID-19 y el mundo del trabajo. Estimaciones actualizadas y análisis. https://www.ilo.org/ wcmsp5/groups/public/---dgreports/--dcomm/documents/briefingnote/ wcms 755917.pdf

Organización para la Cooperación y el Desarrollo Económico- OCDE (2018). Estudios Económicos para América Latina. https://www.cepal. org/sites/default/files/publication/ files/43964/S1800837 es.pdf

Peña, C., Garcés, G., \& Serafim, S. (2020). Los emprendedores zombis: emprendimiento en etapa inicial que no crea empresa en el contexto latinoamericano. Estudios Gerenciales, 36(156), 351-363. https://doi.org/10.18046/j. estger.2020.156.3610

Rodríguez, S., \& Rehner, J. (2021). Emprendimiento en ciudades intermedias en chile: Sus vínculos con el empleo y la sustentabilidad urbana. Revista De Geografía Norte Grande, (78), 93-113. https://doi.org/10.4067/ S0718-34022021000100093

Smyth, E., Gangl, M., Raffe, D., Hannan, D., \& McCony, S. (2003). A comparative analysis of transitions from education to work in Europe. (Catewe): Final Report [and] Annex to the Final Report. Economic and Social Researh Inst. https://www.researchgate. net/publication/234760045 A Comparative Analysis of Transitions from Education to Work in Europe CATEWE Final Report and Annex to the Final Report

Terán, B.M., Valdez, C., \& Miranda, A. (2020). Emprendimiento académico y spin-off universitario: una revisión sistemática de la literatura. Revista Perspectiva Empresarial, 7(1), 87-103. https://doi. org/10.16967/23898186.630

Villalobos-Rodríguez, G., VargasMontero, M., Rodríguez-Ramírez, J., \& Araya-Castillo, L. (2018). Lean start-up as a strategy for the development and management of dynamic entrepreneurships. Dimensión Empresarial, 16(2), 193208. http://dx.doi.org/10.15665/dem. v16i2.1381

Weller, J. (2007, agosto). La inserción laboral de los jóvenes: características, tensiones y desafíos. Revista de La Cepal, 92, 61-82. https://repositorio. cepal.org/handle/11362/11192 